\title{
Quantum Calcium-Ion Affective Influences Measured by EEG
}

\author{
Lester Ingber ${ }^{1}$
}

\begin{abstract}
Background: Since circa 1980, a model of neocortical interactions, Statistical Mechanics of Neocortical Interactions (SMNI) has been successful in calculating many experimental phenomena, including fits to electroencephalographic (EEG) data in attention tasks, using an importance-sampling code Adaptive Simulated Annealing (ASA). The SMNI model is developed in the context of classical path-integrals, which affords intuitive insights as well as direct numerical benefits, e.g., using the effective Action as a a cost/objective function for parameter fits to data. Objective: Previous authors have fit affective EEG data to neural-network models. This project seeks to use models based on physics and biology to fit this same data. Previous work showed improvements in fits to EEG for attention states; this project extends these methods to affective states. Method: Path integrals are used in both classical and quantum contexts. Classical path integrals are used to define a cost/objective function to fit data, and quantum path integrals are used to derive a closed-form analytic expression for Caion waves in the presence of a magnetic vector potential which is generated by highly synchronous neuronal firings which give rise to EEG. ASA is used to fit EEG data. Results: The mathematical-physics and computer parts of the study are successful, in that cost/objective functions used to fit EEG data using these models are consistent with previous work published by other authors. However, since the SMNI model includes these quantum effects, this is another reason to continue examining these issues. The results here are consistent, not better, than previous work using neural-network models, albeit only one parameter was used here, instead of multiple filters and kernels used previously on such data. Conclusion: Although these quantum effects are highly speculative, explicit calculations have shown them to be consistent with experimental data, at least to date. The current supercomputer project extends this model to affective/emotion data. Results from several authors using neural-network approaches at individual electrode sites show some predictive capabilities; the results given here are consistent with these other results. However, since the SMNI model includes these quantum effects, this is another reason to continue examining these issues.
\end{abstract}

Keywords: quantum mechanics; path integral; importance sampling; neuroscience

\section{$1 \quad$ Introduction}

This project builds on previous studies (Ingber, 2018) that have further developed a Statistical Mechanics of Neocortical Interactions (SMNI) by including quantum effects at synaptic gaps.

Over the past decade the author has published a series of papers examining a highly speculative quantum-mechanical neocortical interaction due to experimentally observed Ca-ion waves at tripartite neuron-astrocyte-neuron synaptic sites. Inclusion of this quantum interaction in SMNI was shown to improve fits in EEG taken during attention tasks. The current project reports using this same approach for affective tasks.

Section 2 gives a brief description SMNI.

\footnotetext{
${ }^{1}$ Corresponding author: Lester Ingber

email: lester@ingber.com or ingber@caa.caltech.edu

Additional information may be obtained at https://www.ingber.com.
} 
Section 3 gives a brief description of two algorithms used for computation, path integrals and Adaptive Simulated Annealing (ASA).

Section 4 gives a brief description of the electroencephalographic (EEG) data used in the current study.

Section 4 gives the results of these computations.

Section 5 gives the Conclusion.

\section{$2 \quad$ Statistical Mechanics of Neocortical Interactions (SMNI)}

SMNI scales aggregate synaptic interactions to neuronal firings, up to minicolumnar-macrocolumnar columns of neurons to mesocolumnar dynamics, up to columns of neuronal firings, up to regional macroscopic sites (Ingber, 1981, 1982, 1983, 1984, 1985, 1994).

SMNI has fit experimental data of neocortical interactions, e.g., properties of short-term memory (STM) (Ingber, 2012a), including its capacity (auditory $7 \pm 2$ and visual $4 \pm 2$ ) (Ericsson \& Chase, 1982; Zhang \& Simon, 1985), duration, stability, primacy versus recency rule, as well other phenomenon, e.g., Hick's law (Hick, 1952; Ingber, 1999; Jensen, 1987), interactions within macrocolumns calculating mental rotation of images, etc (Ingber, 1982, 1983, 1984, 1985, 1994). SMNI scaled mesocolumns across neocortical regions to fit EEG data (Ingber, 1997a,b, 2012a).

\section{$2.1 \quad$ Synaptic Interactions}

The short-time conditional probability distribution of firing of a given neuron firing given justprevious firings of other neurons is calculated from chemical and electrical intra-neuronal interactions (Ingber, 1982, 1983). Given its previous interactions with $k$ neurons within $\tau_{j}$ of 5 -10 msec, the conditional probability that neuron $j$ fires $\left(\sigma_{j}=+1\right)$ or does not fire $\left(\sigma_{j}=-1\right)$ is

$$
\begin{gathered}
p_{\sigma_{j}}=\Gamma \Psi=\frac{\exp \left(-\sigma_{j} F_{j}\right)}{\exp \left(F_{j}\right)+\exp \left(-F_{j}\right)} \\
F_{j}=\frac{V_{j}-\sum_{k} a_{j k}^{*} v_{j k}}{\left(\pi \sum_{k^{\prime}} a_{j k^{\prime}}^{*}\left(v_{j k^{\prime}}^{2}+\phi_{j k^{\prime}}^{2}\right)\right)^{1 / 2}} \\
a_{j k}=\frac{1}{2} A_{|j k|}\left(\sigma_{k}+1\right)+B_{j k}
\end{gathered}
$$

The contribution to polarization achieved at an axon given activity at a synapse, taking into account averaging over different neurons, geometries, etc., is given by $\Gamma$, the "intra-neuronal" probability distribution. $\Psi$ is the "inter-neuronal" probability distribution, of thousands of quanta of neurotransmitters released at one neuron's presynaptic site effecting a (hyper-)polarization at another neuron's postsynaptic site, taking into account interactions with neuromodulators, etc. This development holds for $\Gamma$ Poisson, and for $\Psi$ Poisson or Gaussian.

$V_{j}$ is the depolarization threshold in the somatic-axonal region. $v_{j k}$ is the induced synaptic polarization of $E$ or $I$ type at the axon, and $\phi_{j k}$ is its variance. The efficacy $a_{j k}$ is a sum of $A_{j k}$ from the connectivity between neurons, activated if the impinging $k$-neuron fires, and $B_{j k}$ from spontaneous background noise. The efficacy is related to the impedance across synaptic gaps. 


\section{$2.2 \quad$ Neuronal Interactions}

Aggregation up to the mesoscopic scale from the microscopic synaptic scale uses mesoscopic probability $P$

$$
\begin{gathered}
P=\prod_{G} P^{G}\left[M^{G}(r ; t+\tau) \mid M^{\bar{G}}\left(r^{\prime} ; t\right)\right] \\
=\sum_{\sigma_{j}} \delta\left(\sum_{j E} \sigma_{j}-M^{E}(r ; t+\tau)\right) \delta\left(\sum_{j I} \sigma_{j}-M^{I}(r ; t+\tau)\right) \prod_{j}^{N} p_{\sigma_{j}}
\end{gathered}
$$

$M$ represents a mesoscopic scale of columns of $N$ neurons, with subsets $E$ and $I$, represented by $p_{q_{i}} . \bar{G}$ designates chemically independent contributions from both $E$ and $I$.

\section{$2.3 \quad$ Columnar Interactions}

In the prepoint (Ito) representation the SMNI Lagrangian $L$ is

$$
\begin{gathered}
L=\sum_{G, G^{\prime}}(2 N)^{-1}\left(\dot{M}^{G}-g^{G}\right) g_{G G^{\prime}}\left(\dot{M}^{G^{\prime}}-g^{G^{\prime}}\right) /(2 N \tau)-V^{\prime} \\
g^{G}=-\tau^{-1}\left(M^{G}+N^{G} \tanh F^{G}\right) \\
g^{G G^{\prime}}=\left(g_{G G^{\prime}}\right)^{-1}=\delta_{G}^{G^{\prime}} \tau^{-1} N^{G} \operatorname{sech}^{2} F^{G} \\
g=\operatorname{det}\left(g_{G G^{\prime}}\right)
\end{gathered}
$$

The threshold factor $F^{G}$ is derived as

$$
\begin{gathered}
F^{G}=\sum_{G^{\prime}} \frac{\nu^{G}+\nu^{\ddagger E^{\prime}}}{\left((\pi / 2)\left[\left(v_{G^{\prime}}^{G}\right)^{2}+\left(\phi_{G^{\prime}}^{G}\right)^{2}\right]\left(\delta^{G}+\delta^{\ddagger E^{\prime}}\right)\right)^{1 / 2}} \\
\nu^{G}=V^{G}-a_{G^{\prime}}^{G} v_{G^{\prime}}^{G} N^{G^{\prime}}-\frac{1}{2} A_{G^{\prime}}^{G} v_{G^{\prime}}^{G} M^{G^{\prime}}, \nu^{\ddagger E^{\prime}}=-a_{E^{\prime}}^{\ddagger E} v_{E^{\prime}}^{E} N^{\ddagger E^{\prime}}-\frac{1}{2} A_{E^{\prime}}^{\ddagger E} v_{E^{\prime}}^{E} M^{\ddagger E^{\prime}} \\
\delta^{G}=a_{G^{\prime}}^{G} N^{G^{\prime}}+\frac{1}{2} A_{G^{\prime}}^{G} M^{G^{\prime}}, \delta^{\ddagger E^{\prime}}=a_{E^{\prime}}^{\ddagger E} N^{\ddagger E^{\prime}}+\frac{1}{2} A_{E^{\prime}}^{\ddagger E} M^{\ddagger E^{\prime}} \\
a_{G^{\prime}}^{G}=\frac{1}{2} A_{G^{\prime}}^{G}+B_{G^{\prime}}^{G}, a_{E^{\prime}}^{\ddagger E}=\frac{1}{2} A_{E^{\prime}}^{\ddagger E}+B_{E^{\prime}}^{\ddagger E}
\end{gathered}
$$

where $A_{G^{\prime}}^{G}$ is the columnar-averaged direct synaptic efficacy, $B_{G^{\prime}}^{G}$ is the columnar-averaged backgroundnoise contribution to synaptic efficacy. The “" parameters arise from regional interactions across many macrocolumns.

The path integral is derived in terms of mesoscopic Lagrangian $L$. The Action is defined as $L d t$. The "effective Action" is defined as the sum of the Action and the logarithm of the prefactor normalization, and is used as the cost/objective function for fitting data, as this is the true measure of the maximum probability sought by the fitting process. 


\subsection{Tripartite Contributions To Neuronal Firings}

Intercellular calcium waves (ICWs) travel over hundreds of astrocytes affecting many neuronal synapses. ICWs contribute to control synaptic activity (Ross, 2012).

These free regenerative $\mathrm{Ca}^{2+}$ waves arise from astrocyte-neuron interactions, and couple to the magnetic vector potential A produced by highly synchronous collective firings, e.g., during selective attention tasks.

\subsubsection{Canonical Momentum $\Pi=\mathbf{p}+q \mathbf{A}$}

As derived in the Feynman (midpoint) representation of the path integral, the canonical momentum, $\boldsymbol{\Pi}$, defines the dynamics of a moving particle with momentum $\mathbf{p}$ in an electromagnetic field. In SI units,

$$
\boldsymbol{\Pi}=\mathbf{p}+q \mathbf{A}
$$

where $q=-2 e$ for $\mathrm{Ca}^{2+}, e$ is the magnitude of the charge of an electron $=1.6 \times 10^{-19} \mathrm{C}$ (Coulomb), and $\mathbf{A}$ is the electromagnetic vector potential.

\subsubsection{Vector Potential of Wire}

A columnar firing state is modeled as a wire/neuron with current $\mathbf{I}$ measured in $\mathrm{A}=$ Amperes $=$ $\mathrm{C} / \mathrm{s}$,

$$
\mathbf{A}(t)=\frac{\mu}{4 \pi} \int \frac{d r}{r} \mathbf{I}
$$

along a length $z$ observed from a perpendicular distance $r$ from a line of thickness $r_{0}$, yielding

$$
\mathbf{A}=\frac{\mu}{4 \pi} \mathbf{I} \log \left(\frac{r}{r_{0}}\right)
$$

where $\mu$ is the magnetic permeability in vacuum $=4 \pi 10^{-7} \mathrm{H} / \mathrm{m}$ (Henry/meter), which has a log-insensitivity on distance.

\subsubsection{Effects of Vector Potential on Momenta}

The momentum $\mathbf{p}$ for a $\mathrm{Ca}^{2+}$ ion with mass $m=6.6 \times 10^{-26} \mathrm{~kg}$, speed on the order of $50 \mu \mathrm{m} / \mathrm{s}$ to $100 \mu \mathrm{m} / \mathrm{s}$, is on the order of $10^{-30} \mathrm{~kg}-\mathrm{m} / \mathrm{s}$.

The magnitude of the current is taken from experimental data on dipole moments (Murakami \& Okada, 2006; Nunez \& Srinivasan, 2006). Taking $10^{4}$ synchronous firings in a macrocolumn, leads to a dipole moment $|\mathbf{Q}|=10^{-8} \mathrm{~A}-\mathrm{m}$. Taking $z$ to be $10^{2} \mu \mathrm{m}=10^{-4} \mathrm{~m}$, a couple of neocortical layers, gives $|q \mathbf{A}| \approx 2 \times 10^{-19} \times 10^{-7} \times 10^{-8} / 10^{-4}=10^{-28} \mathrm{~kg}-\mathrm{m} / \mathrm{s}$,

\subsubsection{Reasonable Estimates}

Estimates used here for $\mathbf{Q}$ come from experimental data. These include shielding and material effects. When coherent activity among many macrocolumns associated with STM is considered, $|\mathbf{A}|$ may be much larger.

Classical physics calculations give $q \mathbf{A}$ from macroscopic EEG on the order of $10^{-28} \mathrm{~kg}-\mathrm{m} / \mathrm{s}$, while the momentum $\mathbf{p}$ of a $\mathrm{Ca}^{2+}$ ion is on the order of $10^{-30} \mathrm{~kg}-\mathrm{m} / \mathrm{s}$. 
Calculations in classical and quantum physics support the premise that ionic calcium momentumwave effects among neuron-astrocyte-neuron tripartite synapses contribute to background SMNI parameters, thereby creating interactions between ionic/quantum and macroscopic scales (Ingber, 2012a,b, 2015, 2016, 2017a; Ingber et al., 2014; Nunez et al., 2013).

\subsubsection{Quantum Zeno Effects}

The quantum-mechanical wave function of the Ca wave packet was shown to "survive" overlaps after multiple collisions, due to their regenerative processes during the observed long durations of hundreds of ms, leading to the ansatz that $\mathrm{Ca}^{2+}$ waves may support a Zeno or "bang-bang" effect for long coherence times (Burgarth et al., 2018; Facchi et al., 2004; Facchi \& Pascazio, 2008; Giacosa \& Pagliara, 2014; Kozlowski et al., 2015; Muller et al., 2016; Patil et al., 2015; Wu et al., 2012).

Decoherence among particles is very fast, so this premise is highly speculative (Preskill, 2015), but may be determined by experiments.

\subsubsection{Nano-Robotic Applications}

Pharmaceutical products may be transported in nanosystems using this effect (Ingber, 2015), wherein a $\mathrm{Ca}^{2+}$-wave momentum-sensor could act like a piezoelectric device.

\section{Computational Tools}

\subsection{Path Integrals}

Path integral are used here in two ways, for a classical EEG cost function that is used to optimize parameters in a Statistical Mechanics of Interactions (SMNI) model of neocortex, and in for a quantum calculation of a magnetic vector potential contribution to tripartite neuron-astrocyteneuron interactions at synaptic sites.

This project considers interactions across these vast scales using tools of path integrals (Ingber, 2018). Also, while fitting such models to EEG tests some aspects of this project is a somewhat indirect path, but not novel to many physics paradigms that are tested by experiment or computation. It is understood that decoherence is known to be very fast (Preskill, 2015), but unlike other models of neocortex that attempt to directly include quantum effects, the SMNI approach has been supported by direct classical and quantum calculations since 2012 (Ingber, 2012a). Admittedly, it is surprising that detailed calculations continue to support this model, but it is deemed worthwhile for continued examination it until it is theoretically or experimentally proven to be false.

\subsubsection{Classical Path Integrals}

In the context of classical systems, path integrals are used to derive a short-time probability distribution for a two-variable nonlinear (in drifts and diffusions) model of neocortex. This has been established since 1982 in 30+ publications (Ingber, 1982, 1983).

\subsubsection{Quantum Path Integrals}

Codes qPATHINT and qPATHTREE have been developed from their classical counterparts PATHINT and PATHTREE to calculate the propagation of a wave function (Ingber, 2017a b c). 
However, here a closed-form analytic solution is derived for the wave function $\psi_{\mathrm{e}}$ which includes the interaction of $\mathbf{A}$ with $\mathbf{p}$ of $\mathrm{Ca}^{2+}$ wave packets derived from the Feynman representation of the path integral using path-integral techniques (Schulten, 1999), modified here to include A.

$$
\begin{gathered}
\psi_{\mathrm{e}}(t)=\int d \mathbf{r}_{0} \psi_{0} \psi_{F}=\left[\frac{1-i \hbar t /\left(m \Delta \mathbf{r}^{2}\right)}{1+i \hbar t /\left(m \Delta \mathbf{r}^{2}\right)}\right]^{1 / 4}\left[\pi \Delta \mathbf{r}^{2}\left\{1+\left[\hbar t /\left(m \Delta \mathbf{r}^{2}\right)\right]^{2}\right\}\right]^{-1 / 4} \\
\times \exp \left[-\frac{\left[\mathbf{r}-\left(\mathbf{p}_{0}+q \mathbf{A}\right) t / m\right]^{2}}{2 \Delta \mathbf{r}^{2}} \frac{1-i \hbar t /\left(m \Delta \mathbf{r}^{2}\right)}{1+\left[\hbar t /\left(m \Delta \mathbf{r}^{2}\right)\right]^{2}}+i \frac{\mathbf{p}_{0} \cdot \mathbf{r}}{\hbar}-i \frac{\left(\mathbf{p}_{0}+q \mathbf{A}\right)^{2} t}{2 \hbar m}\right] \\
\psi_{F}(t)=\int \frac{d \mathbf{p}}{2 \pi \hbar} \exp \left[\frac{i}{\hbar}\left(\mathbf{p}\left(\mathbf{r}-\mathbf{r}_{0}\right)-\frac{\mathbf{\Pi}^{2} t}{(2 m)}\right)\right]=\left[\frac{m}{2 \pi i \hbar t}\right]^{1 / 2} \exp \left[\frac{i m\left(\mathbf{r}-\mathbf{r}_{0}-q \mathbf{A} t / m\right)^{2}}{2 \hbar t}-\frac{i(q \mathbf{A})^{2} t}{2 m \hbar}\right] \\
\psi_{0}=\psi\left(\mathbf{r}_{0}, t=0\right)=\left(\frac{1}{\pi \Delta \mathbf{r}^{2}}\right)^{1 / 4} \exp \left(-\frac{\mathbf{r}_{0}^{2}}{2 \Delta \mathbf{r}^{2}}+i \frac{\mathbf{p}_{0} \cdot \mathbf{r}_{0}}{\hbar}\right)
\end{gathered}
$$

$\psi_{0}$ is the initial Gaussian packet, $\psi_{F}$ is the free-wave evolution operator, $\hbar$ is the Planck constant, $q$ is the electronic charge of $\mathrm{Ca}^{2+}$ ions, $m$ is the mass of a wave-packet of $1000 \mathrm{Ca}^{2+}$ ions, $\Delta \mathbf{r}^{2}$ is the spatial variance of the wave-packet, the initial momentum is $\mathbf{p}_{0}$, and the evolving canonical momentum is $\boldsymbol{\Pi}=\mathbf{p}+q \mathbf{A}$. Calculations show $\mathbf{p}$ of the $\mathrm{Ca}^{2+}$ wave packet and $q \mathbf{A}$ of the EEG field make about equal contributions to $\boldsymbol{\Pi}$ (Ingber, 2015).

Tripartite influence on synaptic $B_{G^{\prime}}^{G}$ is calculated by the ratio of packet's $<\mathbf{p}(t)>_{\psi * \psi}$ to $<\mathbf{p}_{0}\left(t_{0}\right)>_{\psi * \psi}$ at the onset of each attentional task. Here $<>_{\psi * \psi}$ is taken over $\psi_{\mathrm{e}}^{*} \psi_{\mathrm{e}}$.

$$
<\mathbf{p}>_{\psi * \psi}=m \frac{<\mathbf{r}>_{\psi * \psi}}{t-t_{0}}=\frac{q \mathbf{A}+\mathbf{p}_{0}}{m^{1 / 2}|\Delta \mathbf{r}|}\left(\frac{(\hbar t)^{2}+\left(m \Delta \mathbf{r}^{2}\right)^{2}}{\hbar t+m \Delta \mathbf{r}^{2}}\right)^{1 / 2}
$$

A changes slower than $\mathbf{p}$, so static approximation of $\mathbf{A}$ used to derive $\psi_{\mathrm{e}}$ and $\left.<\mathbf{p}\right\rangle_{\psi * \psi}$ is reasonable to use within EEG epochs, resetting $t=0$ at the onset of each classical EEG measurement, using the current $\mathbf{A}$.

The $\hbar t$-dependence of this result makes it possible to experimentally test these effects, e.g., in EEG fits as well as perhaps more directly in future experiments.

\subsection{Adaptive simulated annealing (ASA)}

This ASA algorithm is faster than fast Cauchy annealing, which has schedule $T_{i}=T_{0} / k$, and much faster than Boltzmann annealing, which has schedule $T_{i}=T_{0} / \ln k(\overline{I n g b e r}, 1989)$.

\subsection{Outline of ASA Algorithm}

For parameters

$$
\alpha_{k}^{i} \in\left[A_{i}, B_{i}\right]
$$

sampling with the random variable $x^{i}$

$$
\begin{gathered}
x^{i} \in[-1,1] \\
\alpha_{k+1}^{i}=\alpha_{k}^{i}+x^{i}\left(B_{i}-A_{i}\right)
\end{gathered}
$$


the default generating function is

$$
g_{T}(x)=\prod_{i=1}^{D} \frac{1}{2 \ln \left(1+1 / T_{i}\right)\left(\left|x^{i}\right|+T_{i}\right)} \equiv \prod_{i=1}^{D} g_{T}^{i}\left(x^{i}\right)
$$

in terms of parameter "temperatures"

$$
T_{i}=T_{i 0} \exp \left(-c_{i} k^{1 / D}\right)
$$

The default ASA uses the same type of annealing schedule for the acceptance function $h$ as used for the generating function $g$.

All default functions in ASA can be overridden with user-defined functions.

\section{Data Sources: GAMEEMO}

The EEG data used for this study was made publicly available via Mendeley from a study of emotion recognition on 4 types of computer games (Alakus et al., 2020). The computer games consist of 4 types of affective behavior:

Game 1: low arousal negative valence (LANV), e.g., sad, bored, sleepy.

Game 2: low arousal positive valence (LAPV), e.g., calm, relaxed.

Game 3: high arousal negative valence (HANV), e.g., nervous, anger.

Game 4: high arousal positive valence (HAPV), e.g., pleased, excited.

The data generated was confirmed by detailed questionnaires designed to confirm or deny the affective states assumed by the experimental paradigms.

These emotional states were measured over durations of several minutes, and typically they do not produce large EEG peaks, e.g., such as those associated with attention states which have fractions of seconds of duration. Thus, the SMNI model had much more difficulty fitting affective data than it had done previously with attention data.

The data collection required 1,568 Training runs over 14 Electrode sites, 28 Subjects and 4 Games. Each Training run had 4 subsequent Testing runs, one for each Game. The lowest cost function (effective Action) of Training sets per Electrode per Subject per Game determined whether or not there was a match between the Training set and the 4 subsequent Testing sets. Electrode sites selected were $\{$ AF3, AF4, F3, F4, F7, F8, FC5, FC6, O1, O2, P7, P8, T7, T8\}.

Since the subjects physically moved during these games, enhancing their affective states, the data was pre-cleaned for movement artifacts, making it even more useful for research.

\section{$5 \quad$ Results}

Only one parameter, multiplying the synaptic background from a rage [0.1,0.5], was used for ASAfitting EEG for each electrode, followed up by simplex fits which did not improve any results. In all previous SMNI studies fitting EEG, all electrode sources were used and parameters $\mathrm{n}$ each region 
weighted contributions from other regions. The parameter selected for this study weights the scale mapping the EEG data into neuronal firing space, also proportional to experimentally observed maximum number of neurons per minicolumn which is twice that in visual neocortex than in other regions.

The authors of the data used multiple trial-and-error parameters in their neural-network calculations, as well as the use of several classification filters and kernels (Alakus et al., 2020).

The percent of success over all 28 Subjects for each Game is given in Fig. 1, where column 1 is the percent $(\mathrm{P})$ correct match between the Training cost function and the lowest Testing cost function over all 28 Subjects; column 2 the game $(G)$ number; column 3 is the electrode (E) site. 


\begin{tabular}{|lll|} 
P & G & E \\
\hline \hline 0.607 & 4 & T8 \\
0.607 & 4 & F8 \\
0.571 & 4 & FC6 \\
0.535 & 4 & T7 \\
0.500 & 4 & F7 \\
0.500 & 4 & AF4 \\
0.428 & 4 & F4 \\
0.428 & 4 & F3 \\
0.428 & 1 & O2 \\
0.392 & 4 & P7 \\
0.392 & 4 & AF3 \\
0.357 & 4 & P8 \\
0.321 & 4 & O1 \\
0.321 & 3 & O1 \\
0.321 & 1 & O1 \\
0.285 & 4 & O2 \\
0.285 & 4 & FC5 \\
0.285 & 3 & T8 \\
0.285 & 3 & AF4 \\
0.214 & 2 & O2 \\
0.214 & 1 & T7 \\
0.214 & 1 & P8 \\
0.178 & 2 & F3 \\
0.178 & 2 & AF3 \\
0.178 & 1 & P7 \\
0.142 & 3 & T7 \\
0.142 & 3 & FC6 \\
0.142 & 3 & FC5 \\
0.142 & 2 & F7 \\
0.142 & 2 & F4 \\
0.142 & 1 & FC6 \\
0.142 & 1 & FC5 \\
0.142 & 1 & F7 \\
0.142 & 1 & AF3 \\
0.107 & 3 & F8 \\
0.107 & 3 & F4 \\
0.107 & 3 & F3 \\
0.107 & 1 & F8 \\
0.071 & 3 & P8 \\
0.071 & 3 & P7 \\
0.071 & 3 & O2 \\
0.071 & 2 & P8 \\
0.071 & 2 & O1 \\
0.071 & 1 & T8 \\
0.071 & 1 & F4 \\
0.071 & 1 & F3 \\
0.071 & 1 & AF4 \\
0.035 & 2 & T7 \\
0.035 & 2 & FC6 \\
0.035 & 2 & AF4 \\
0.000 & 3 & F7 \\
0.000 & 3 & AF3 \\
0.000 & 2 & T8 \\
0.000 & 2 & P7 \\
0.000 & 2 & FC5 \\
0.000 & 2 & F8 \\
& &
\end{tabular}

Figure 1. Column 1 is the percent $(\mathrm{P})$ correct match between the Training cost function and the lowest Testing cost function over all 28 Subjects. Column 2 is the Game $(\mathrm{G})$ number. Column 3 is the electrode (E) site.

Epochs of duration 1/128 for each electrode site varied, but were always over 8000. Data used were from epoch 2000 through 6000. Since their was just one session per Subject per Game, Training sets were determined by selecting every even data for Training and every odd data for Testing, resulting in an effective $d t$ duration of 2/128 for each Training/Testing set of 1000 epochs. 
These results are consistent, but not as good, with those obtained for single electrodes by the authors of the data (Alakus et al., 2020), as verified in a private email with the lead author. However, here the model includes quantum effects from tripartite influences.

\section{Conclusion}

A model of neocortical interactions, Statistical Mechanics of Neocortical Interactions (SMNI) has been successful in calculating many experimental phenomena, including fits to EEG data in attention tasks, using an importance-sampling code Adaptive Simulated Annealing (ASA). The SMNI model is developed in the context of classical path-integrals, which affords intuitive insights as well as direct numerical benefits, e.g., using the effective Action as a a cost/objective function for parameter fits to data.

Results from several authors using neural-network approaches at individual electrode sites, show some predictive capabilities; the results given here are consistent with these other results. However, since the SMNI model includes these quantum effects, this is another reason to continue examining these issues.

\section{Acknowledgment}

The author thanks the Extreme Science and Engineering Discovery Environment (XSEDE.org), for supercomputer grants since February 2013. XSEDE grants have spanned several projects described in https://www.ingber.com/lir_computational_physics_group.html . 


\section{References}

Alakus, T., Gonen, M., Turkoglu, I. (2020). Database for an emotion recognition system based on EEG signals andvarious computer games - GAMEEMO. Biomedical Signal Processing and Control 60(101951), 1-12. https://doi.org/10.1016/j.bspc.2020.101951.

Burgarth, D., Facchi, P., Nakazato, H., Pascazio, S., Yuasa, K. (2018). Quantum zeno dynamics from general quantum operations. Technical Report arXiv:1809.09570v1 [quant-ph], Aberystwyth U., Aberystwyth, UK.

Ericsson, K., Chase, W. (1982). Exceptional memory. American Scientist 70, 607-615.

Facchi, P., Lidar, D., Pascazio, S. (2004). Unification of dynamical decoupling and the quantum zeno effect. Physical Review A 69(032314), 1-6.

Facchi, P., Pascazio, S. (2008). Quantum zeno dynamics: mathematical and physical aspects. Journal of Physics A 41(493001), 1-45.

Giacosa, G., Pagliara, G. (2014). Quantum zeno effect by general measurements. Physical Review A 052107, 1-5.

Hick, W. (1952). On the rate of gains of information. Quarterly Journal Experimental Psychology $34(4), 1-33$.

Ingber, L. (1981). Towards a unified brain theory. Journal Social Biological Structures 4, 211-224. https://www.ingber.com/smni81_unified.pdf.

Ingber, L. (1982). Statistical mechanics of neocortical interactions. i. basic formulation. Physica D 5, 83-107. https://www.ingber.com/smni82_basic.pdf.

Ingber, L. (1983). Statistical mechanics of neocortical interactions. dynamics of synaptic modification. Physical Review A 28, 395-416. https://www.ingber.com/smni83_dynamics.pdf.

Ingber, L. (1984). Statistical mechanics of neocortical interactions. derivation of short-term-memory capacity. Physical Review A 29, 3346-3358. https://www.ingber.com/smni84_stm.pdf.

Ingber, L. (1985). Statistical mechanics of neocortical interactions: Stability and duration of the 7+-2 rule of short-term-memory capacity. Physical Review A 31, 1183-1186. https://www. ingber.com/smni85_stm.pdf.

Ingber, L. (1989). Very fast simulated re-annealing. Mathematical Computer Modelling 12(8), 967-973. https://www.ingber.com/asa89_vfsr.pdf.

Ingber, L. (1994). Statistical mechanics of neocortical interactions: Path-integral evolution of shortterm memory. Physical Review E 49(5B), 4652-4664. https://www.ingber.com/smni94_stm. pdf.

Ingber, L. (1997a). Statistical mechanics of neocortical interactions: Applications of canonical momenta indicators to electroencephalography. Physical Review E 55(4), 4578-4593. https: //www.ingber.com/smni97_cmi.pdf 
Ingber, L. (1997b). EEG Database. UCI Machine Learning Repository, Irvine, CA. http:// archive.ics.uci.edu/ml/datasets/EEG+Database.

Ingber, L. (1999). Statistical mechanics of neocortical interactions: Reaction time correlates of the g factor. Psycholoquy 10(068). Invited commentary on The g Factor: The Science of Mental Ability by Arthur Jensen. http://www.cogsci.ecs.soton.ac.uk/cgi/psyc/newpsy?10.068.

Ingber, L. (2012a). Columnar EEG magnetic influences on molecular development of short-term memory. In Kalivas, G., Petralia, S. (Eds.), Short-Term Memory: New Research, Nova, Hauppauge, NY. 37-72. Invited Paper. https://www.ingber.com/smni11_stm_scales.pdf.

Ingber, L. (2012b). Influence of macrocolumnar EEG on ca waves. Current Progress Journal 1(1), 4-8. https://www.ingber.com/smni12_vectpot.pdf

Ingber, L. (2015). Calculating consciousness correlates at multiple scales of neocortical interactions. In Costa, A., Villalba, E. (Eds.), Horizons in Neuroscience Research, Nova, Hauppauge, NY. 153-186. ISBN 978-1-63482-632-7. Invited paper. https://www.ingber.com/smni15_calc_ conscious.pdf.

Ingber, L. (2016). Statistical mechanics of neocortical interactions: Large-scale EEG influences on molecular processes. Journal of Theoretical Biology 395, 144-152. https://dx.doi.org/10. $1016 / \mathrm{j} \cdot \mathrm{jtbi} .2016 .02 .003$.

Ingber, L. (2017a). Evolution of regenerative ca-ion wave-packet in neuronal-firing fields: Quantum path-integral with serial shocks. International Journal of Innovative Research in Information Security 4(2), 14-22. https://www.ingber.com/path17_quantum_pathint_shocks.pdf.

Ingber, L. (2017b). Options on quantum money: Quantum path-integral with serial shocks. International Journal of Innovative Research in Information Security 4(2), 7-13. https: //www.ingber.com/path17_quantum_options_shocks.pdf.

Ingber, L. (2017c). Quantum path-integral qPATHINT algorithm. The Open Cybernetics Systemics Journal 11, 119-133. https://dx.doi.org/10.2174/1874110X01711010119.

Ingber, L. (2018). Quantum calcium-ion interactions with EEG. Sci 1(7), 1-21. https://doi. org/10.3390/sci1010020.

Ingber, L., Pappalepore, M., Stesiak, R. (2014). Electroencephalographic field influence on calcium momentum waves. Journal of Theoretical Biology 343, 138-153. https://dx.doi.org/10.1016/ j.jtbi.2013.11.002.

Jensen, A. (1987). Individual differences in the hick paradigm. In Vernon, P. (Ed.), Speed of Information-Processing and Intelligence, Ablex, Norwood, NJ. 101-175.

Kozlowski, W., Caballero-Benitez, S., Mekhov, I. (2015). Non-hermitian dynamics in the quantum zeno limit. Technical Report arXiv:1510.04857 [quant-ph], U Oxford, Oxford, UK.

Muller, M., Gherardini, S., Caruso, F. (2016). Quantum zeno dynamics through stochastic protocols. Technical Report arXiv:1607.08871v1 [quant-ph], U Florence, Florence, Italy.

Murakami, S., Okada, Y. (2006). Contributions of principal neocortical neurons to magnetoencephalography and electroencephalography signals. Journal of Physiology 575(3), 925-936. 
Nunez, P., Srinivasan, R. (2006). Electric Fields of the Brain: The Neurophysics of EEG, 2nd Ed. Oxford University Press, London.

Nunez, P., Srinivasan, R., Ingber, L. (2013). Theoretical and experimental electrophysiology in human neocortex: Multiscale correlates of conscious experience. In Pesenson, M. (Ed.), Multiscale Analysis and Nonlinear Dynamics: From genes to the brain, Wiley, New York. 149-178. https: //dx.doi.org/10.1002/9783527671632.ch06.

Patil, Y., Chakram, S., Vengalattore, M. (2015). Measurement-induced localization of an ultracold lattice gas. Physical Review Letters 115(140402), 1-5. http://link.aps.org/doi/10.1103/ PhysRevLett.115.140402.

Preskill, J. (2015). Quantum mechanics. Technical Report Lecture Notes, Caltech, Pasadena, CA. http://www.theory.caltech.edu/people/preskill/ph219/.

Ross, W. (2012). Understanding calcium waves and sparks in central neurons. Nature Reviews Neuroscience 13, 157-168.

Schulten, K. (1999). Quantum mechanics. Technical Report Lecture Notes, U. Illinois, Urbana, IL. http://www.ks.uiuc.edu/Services/Class/PHYS480/

Wu, S., Wang, L., Yi, X. (2012). Time-dependent decoherence-free subspace. Journal of Physics A 405305, 1-11.

Zhang, G., Simon, H. (1985). STM capacity for chinese words and idioms: Chunking and acoustical loop hypotheses. Memory and Cognition 13, 193-201. 